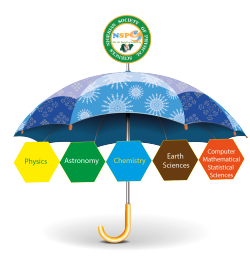

\title{
Application of the Exponentiated Log-Logistic Weibull Distribution to Censored Data
}

\author{
Adeniyi F. Fagbamigbe ${ }^{\mathrm{a}, *}$, Gomolemo K. Basele ${ }^{\mathrm{b}}$, Boikanyo Makubate ${ }^{\mathrm{b}}$, Broderick O. Oluyede \\ ${ }^{a}$ Department of Epidemiology and Medical Statistics, College of Medicine, Faculty of Public Health, University of Ibadan, Nigeria \\ ${ }^{b}$ Department of Mathematics and Statistical Sciences, Botswana International University of Science and Technology, Palapye, BW \\ ${ }^{c}$ Department of Mathematical Sciences, Georgia Southern University, Statesboro, GA, 30460, USA
}

\begin{abstract}
In a recent paper, a new model called the Exponentiated Log-Logistic Weibull (ELLoGW) distribution with applications to reliability, survival analysis and income data was proposed. In this study, we applied the recently developed ELLoGW model to a wide range of censored data. We found that the ELLoGW distribution is a very competitive model for describing censored observations in life-time reliability problems such as survival analysis. This work shows that in certain cases, the ELLoGW distribution performs better than other parametric model such as the Log-Logistic Weibull, Exponentiated Log-Logistic Exponential, Log-Logistic Exponential distributions and the non-nested Gamma-Dagum (GD).
\end{abstract}

Keywords: Generalized Distribution, Exponentiated log-logistic Distribution, Weibull Distribution, Survival Analysis.

Article History :

Received: 31-03-2019

Received in revised form: 23-04-2019

Accepted for publication: 23-04-2019

Published: 26-04-2019

(C)2019 Journal of the Nigerian Society of Physical Sciences. All Rights Reserved. Communicated by: B. J. Falaye

\section{Introduction}

Oluyede et al. had recently proposed a new model called the Exponentiated Log-Logistic Weibull (ELLoGW) distribution with applications to reliability, survival analysis and income data [1]. In this paper, we are primarily concerned with the application of the new model (ELLoGW) to censored data. The details of the model and all its associated properties including the hazard function, reverse hazard function, quantile function, moments, conditional moments, mean deviations, Bonferroni and Lorenz curves, entropy and order statistics have been reported earlier [1].

Censored data arise when a study comes to an end before every subject been studied experience the event of interest $[2$,

\footnotetext{
${ }^{*}$ Corresponding Author Tel. No: +2348061348165

Email address: fadeniyi@cartafrica.org (Adeniyi F. Fagbamigbe )
}

3]. In this study we applied data collected in situations where an experimentalist involved $n$ samples in a life-testing experiment. The samples were kept under observation until "failure" occur. The samples could be electrical or mechanical components, system, individuals, or perhaps computer chips in a reliability experiment.

It could also be patients, subjects or individuals that are recruited for drug or clinical trials. In most cases, such experiments are terminated before all the samples could have "failed". The same could be said of drug or clinical trials wherein the recruited subjects might have been lost to follow up, dropped out of the study, withdrew from the study or the study may come to an end due to unforeseen circumstances or economic conditions or exhausted time frame.

Also, samples could break accidentally, malfunction in an industrial experiment and thus referred to as "failure". Generally, the data obtained under these or similar scenarios are 
called censored data. Literature is replete on application of statistical distribution to different types of data $[1,4,5,6]$. A brief review of the model, the different types of censored data and the application of the model to different types of censored data are discussed in the subsequent sections.

\section{The ELLoGW Distribution}

In this section, the generalized or exponentiated log-logistic Weibull (ELLoGW) distribution is presented. The generalized or exponentiated log-logistic Weibull (ELLoGW) (see Oluyede et al. [1] for details) distribution function is given by

$$
G_{E L L O G W}(x)=\left[1-\left(1+\left(\frac{x}{s}\right)^{c}\right)^{-1} \exp \left(-\alpha x^{\beta}\right)\right]^{\delta},
$$

for $s, c, \alpha, \beta, \delta$, and $x \geq 0$. The corresponding probability density function (pdf) is given by

$$
\begin{aligned}
g_{E L L O G W}(x)= & \delta\left[1-\left(1+\left(\frac{x}{s}\right)^{c}\right)^{-1} \exp \left(-\alpha x^{\beta}\right)\right]^{\delta-1} e^{-\alpha x^{\beta}} \\
& \times\left[1+\left(\frac{x}{s}\right)^{c}\right]^{-1}\left\{\alpha \beta x^{\beta-1}+\frac{c x^{c-1}}{\left(s^{c}+x^{c}\right)}\right\},
\end{aligned}
$$

for $s, c, \alpha, \beta, \delta>0$, and $x \geq 0$. The corresponding hazard function is given by

$$
\begin{aligned}
h_{E L L O G W}(x)= & \delta\left[1-\left(1+\left(\frac{x}{s}\right)^{c}\right)^{-1} e^{-\alpha x^{\beta}}\right]^{\delta-1} \\
& \times e^{-\alpha x^{\beta}}\left[1+\left(\frac{x}{s}\right)^{c}\right]^{-1}\left\{\alpha \beta x^{\beta-1}+\frac{c x^{c-1}}{\left(s^{c}+x^{c}\right)}\right\} \\
& \times\left[1-\left\{1-\left(1+\left(\frac{x}{s}\right)^{c}\right)^{-1} \exp \left(-\alpha x^{\beta}\right)\right\}^{\delta}\right]^{-1} .
\end{aligned}
$$

\section{Censoring}

In the following section we construct log-likelihood functions of the ELLoGW distribution to deal with type I right censoring and type II doubly censored observations.

\subsection{Type I Right Censoring}

This is the most common form of incomplete data often encountered in survival analysis. Type I censoring arises when the study is conducted over a specified time period that can terminate before all the units have failed. Each individual has a fixed censoring time $C_{i}$, which would be the time between the date of entry and the end of the study, so that the complete failure time of an individual will be known only if it is less than or equal to the censoring time $T_{i} \leq C_{i}$; otherwise, only a lower bound of the individual lifetime is available $T_{i}>C_{i}$. The data for this design are conveniently indicated by pairs of random variables $\left(T_{i}, \varepsilon_{i}\right), i=1, \ldots, n$. Consider a sample of size $\mathrm{n}$ of independent positive random variables $T_{1}, \ldots, T_{n}$, such that $T_{i}$ is associated with an indicator variable $\varepsilon_{i}=0$ if $T_{i}$ is a censoring time. Let $\Theta=(\mathrm{s}, \mathrm{c}, \alpha, \beta, \delta)^{T}$, then the likelihood function, $L(\Theta)$, of a type I right censored sample $\left(t_{1}, \varepsilon_{1}\right), \ldots,\left(t_{n}, \varepsilon_{n}\right)$ from the ELLoGW distribution with pdf $g_{E L L O G W}($.$) and survival function$ $S_{E L L o G W}($.) can be written as

$$
L(\underline{\Theta})=\prod_{i=1}^{n} g_{E L L o G W}\left(t_{i}\right)^{\epsilon_{i}} S_{E L L o G W}\left(t_{i}\right)^{1-\epsilon_{i}},
$$

where $S_{E L L o G W}\left(t_{i}\right)=1-G_{E L L o G W}\left(t_{i}\right)$. The log-likelihood function, $l(\Theta)$, based on data, from the ELLoGW distribution is,

$$
\begin{aligned}
l(\underline{\Theta})= & \sum_{i=1}^{n}\left\{\epsilon _ { i } \operatorname { l n } \left[\delta\left(1-\left(1+\left(\frac{t_{i}}{s}\right)^{c}\right)^{-1} e^{-\alpha t_{i}^{\beta}}\right)^{\delta-1}\right.\right. \\
& \left.\times e^{-\alpha t_{i}^{\beta}}\left(1+\left(\frac{t_{i}}{s}\right)^{c}\right)^{-1}\left(\alpha \beta t_{i}^{\beta-1}+\frac{c t_{i}^{c-1}}{s^{c}+t_{i}^{c}}\right)\right] \\
& \left.+\left(1-\epsilon_{i}\right) \ln \left[1-\left(1-\left(1+\left(\frac{t_{i}}{s}\right)^{c}\right)^{-1} e^{-\alpha t_{i}^{\beta}}\right)^{\delta}\right]\right\} .
\end{aligned}
$$

Elements of the score vector are given by

$$
\left(\frac{\partial l(\underline{\Theta})}{\partial s}, \frac{\partial l(\underline{\Theta})}{\partial c}, \frac{\partial l(\underline{\Theta})}{\partial \alpha}, \frac{\partial l(\underline{\Theta})}{\partial \beta}, \frac{\partial l(\underline{\Theta})}{\partial \delta}\right)^{T} .
$$

The MLEs $\underline{\hat{\Theta}}=(\hat{s}, \hat{c}, \hat{\alpha}, \hat{\beta}, \hat{\delta})^{T}$ are obtained from the numerical maximization of equation (4). Let $\underline{\Theta}=(s, c, \alpha, \beta, \delta)^{T}$ be the parameter vector and $\underline{\hat{\Theta}}=(\hat{s}, \hat{c}, \hat{\alpha}, \hat{\beta}, \hat{\delta})^{T}$ be the maximum likelihood estimate of $\bar{\Theta}=(s, c, \alpha, \beta, \delta)^{T}$. Under the usual regularity conditions and that the parameters are in the interior of the parameter space, but not on the boundary, [12] we have: $\sqrt{n}(\underline{\hat{\Theta}}-\underline{\Theta}) \underset{d}{\rightarrow} N_{5}\left(\underline{0}, I^{-1}(\underline{\Theta})\right)$, where $\mathrm{I}(\underline{\Theta})$ is the expected Fisher Information Matrix. The asymptotic behavior is still valid if $\mathrm{I}(\Theta)$ is replaced by the observed information matrix evaluated at $\underline{\hat{\Theta}}$, that is $\mathrm{J}(\underline{\hat{\Theta}})$. The multivariate normal distribution $N_{5}\left(\underline{0}, J(\underline{\hat{\Theta}})^{-1}\right)$, where the mean vector $\underline{0}=(0,0,0,0,0)^{T}$, can be used to construct confidence intervals and confidence regions for the individual model parameters and for the survival and hazard functions.

\subsection{Type II Censoring}

For type II censoring, the data consists of the $r^{\text {th }}$ smallest lifetimes $X_{(1)} \leq X_{(2)} \leq \ldots \leq X_{(r)}$ out of a random sample of $\mathrm{n}$ lifetimes $X_{1}, X_{2}, \ldots, X_{n}$ from the ELLoGW distribution. Assuming $X_{1} \leq X_{2} \leq \ldots \leq X_{n}$ are independent and identically distributed and have a continuous distribution with pdf $g_{E L L O G W}($.) and survival function $S_{E L L O G W}($.$) , it follows that the joint pdf of$ $X_{(1)}, \ldots, X_{(r)}$ is

$$
L(\underline{\Theta})=\frac{n !}{(n-r) !}\left[\prod_{i=1}^{r} g_{E L L o G W}\left(x_{(i)}\right)\right]\left[S_{E L L o G W}\left(x_{(r)}\right)\right]^{n-r},
$$


Table 1: Times to Infection Data

\begin{tabular}{|c|c|c|c|c|c|c|c|c|c|c|c|c|c|}
\hline \multirow[b]{2}{*}{ Model } & \multicolumn{5}{|c|}{ Estimates } & \multicolumn{8}{|c|}{ Statistics } \\
\hline & $s$ & $c$ & $\alpha$ & $\beta$ & $\delta$ & $-2 \log L$ & $A I C$ & $A I C C$ & $B I C$ & $W^{*}$ & $A^{*}$ & KS pvalue & $S S$ \\
\hline ELLoGW & $\begin{array}{c}2.1674 \\
(2.5612)\end{array}$ & $\begin{array}{c}0.8092 \\
(0.7057)\end{array}$ & $\begin{array}{c}0.0090 \\
(0.0450)\end{array}$ & $\begin{array}{c}1.7083 \\
(1.4104)\end{array}$ & $\begin{array}{c}4.1106 \\
(2.9263)\end{array}$ & 98.0372 & 108.0372 & 114.7039 & 111.5775 & 0.0225 & 0.1556 & 0.999 & 0.0196 \\
\hline LLoGW & $\begin{array}{l}20.4723 \\
(0.0309)\end{array}$ & $\begin{array}{c}1.5446 \\
(1.0376)\end{array}$ & $\begin{array}{c}0.0071 \\
(0.0193)\end{array}$ & $\begin{array}{c}1.7720 \\
(0.8868)\end{array}$ & $\begin{array}{l}1 \\
-\end{array}$ & 98.2032 & 106.2032 & 112.8698 & 109.0354 & 0.0244 & 0.1655 & 0.9988 & 0.0213 \\
\hline ELLoGE & $\begin{array}{c}0.0192 \\
(0.0991)\end{array}$ & $\begin{array}{c}0.3982 \\
(0.4326)\end{array}$ & $\begin{array}{c}0.1266 \\
(0.0633)\end{array}$ & $\begin{array}{l}1 \\
-\end{array}$ & $\begin{array}{l}28.8554 \\
(0.0002)\end{array}$ & 98.4424 & 106.4424 & 113.1091 & 109.2746 & 0.0242 & 0.1697 & 0.9922 & 0.0208 \\
\hline LLoGE & $\begin{array}{l}12.5296 \\
(7.2980)\end{array}$ & $\begin{array}{c}2.5301 \\
(1.0222)\end{array}$ & $\begin{array}{c}0.0295 \\
(0.0482)\end{array}$ & $\begin{array}{l}1 \\
-\end{array}$ & $\begin{array}{l}1 \\
-\end{array}$ & 99.4833 & 105.4833 & 112.1500 & 107.6075 & 0.0283 & 0.1963 & 0.9981 & 0.0193 \\
\hline GD & $\begin{array}{c}\lambda \\
9.0005 \\
(13.2476)\end{array}$ & $\begin{array}{c}\beta \\
1.9151 \\
(2.5511)\end{array}$ & $\begin{array}{c}\delta \\
0.4786 \\
(0.4362)\end{array}$ & $\begin{array}{c}\alpha \\
3.9043 \\
(3.4708)\end{array}$ & $\begin{array}{c}\theta \\
0.0192 \\
(0.0658)\end{array}$ & 98.7984 & 108.7984 & 115.4651 & 112.3386 & 0.0274 & 0.1932 & 0.9676 & 0.0254 \\
\hline
\end{tabular}

where $S_{E L L o G W}(x)=1-G_{E L L o G W}(x)$. The log-likelihood function, $l(\Theta)$, based on data from the ELLoGW distribution is,

$$
\begin{aligned}
l(\underline{\Theta})= & \ln \left[\frac{n !}{(n-r) !}\right]+\sum_{i=1}^{r}\{\ln \delta+(\delta-1) \\
& \times \ln \left[1-\left(1+\left(\frac{x_{(i)}}{s}\right)^{c}\right)^{-1} e^{-\alpha x_{(i)}^{\beta}}\right]-\alpha x_{(i)}^{\beta} \\
& -\ln \left[1+\left(\frac{x_{(i)}}{s}\right)^{c}\right]+\ln \left\{\alpha \beta x_{(i)}^{\beta-1}+\frac{c x_{(i)}^{c-1}}{s^{c}+x_{(i)}^{c}}\right\} \\
& \left.+(n-r) \ln \left[1-\left(1-\left(1+\left(\frac{x_{\left(r_{1}\right)}}{s}\right)^{c}\right) e^{-\alpha x_{\left(r_{1}\right)}^{\beta}}\right)^{\delta}\right]\right\} .
\end{aligned}
$$

Elements of the score vector and MLE's are similar to those in the type I right censoring scheme.

\subsection{Type II Double Censoring}

Type II double censoring is a censoring procedure wherein a fixed number of observations is missing at the two ends of a n sample size unlike in type I censoring, where the number of censored observations is totally random and the study time is predetermined. The data consist of the remaining ordered observations $t_{r+1}, \ldots, t_{m}$ when the $r$ smallest observations and the $n-m$ largest observations are out of a sample of size $\mathrm{n}$ from the ELLoGW distribution. The likelihood function, $L(\underline{\Theta})$, of the type II doubly censored sample $t_{(r+1)}, \ldots, t_{(m)}$ from the ELLoGW distribution with pdf $g_{E L L O G W}($.$) , cdf G_{E L L o G W}($.$) and survival$ function $S_{E L L O G W}($.) is given by

$$
\begin{aligned}
L(\underline{\Theta})= & \frac{n !}{r !(n-m) !}\left\{G_{E L L o G W}\left(t_{(r+1)}\right)\right\}^{r} \\
& \times\left\{S_{E L L o G W}\left(t_{(m)}\right)\right\}^{n-m} \prod_{i=r+1}^{m} g_{E L L o G W}\left(t_{(i)}\right) .
\end{aligned}
$$

The log-likelihood function, $l(\Theta)$, for a type II doubly censored sample $t_{(r+1)}, \ldots t_{(m)}$ from the ELLoGW distribution is given by

$$
\begin{aligned}
l(\underline{\Theta})= & \ln \left(\frac{n !}{r !(n-m) !}\right) \\
& +r \ln \left[\left(1-\left(1+\left(\frac{t_{(r+1)}}{s}\right)^{c}\right)^{-1} e^{-\alpha t_{(r+1)^{\beta}}}\right)^{\delta}\right] \\
& +(n-m) \ln \left[1-\left(1-\left(1+\left(\frac{t_{(m)}}{s}\right)^{c}\right)^{-1} e^{-\alpha t_{(m)}^{\beta}}\right)^{\delta}\right] \\
& +\sum_{i=r+1}^{m} \ln \left\{\delta\left(1-\left(1+\left(\frac{t_{(i)}}{s}\right)^{c}\right)^{-1} e^{-\alpha t_{(i)}^{\beta}}\right)^{\delta-1}\right. \\
& \left.\times e^{-\alpha t_{(i)}^{\beta}}\left(1+\left(\frac{t_{(i)}}{s}\right)^{c}\right)^{-1}\left(\alpha \beta t_{(i)}^{\beta-1}+\frac{c t_{(i)}^{c-1}}{s^{c}+t_{(i)}^{c}}\right)\right\} .
\end{aligned}
$$

Elements of the score vector and their MLE's are similar to those in the type I right censoring scheme.

\section{Applications}

In this section, we give some applications to real life data. Maximum likelihood estimates of the model parameters under type I right and type II double censored data are obtained and comparisons with the Log-Logistic Weibull (LLoGW), Exponentiated Log-Logistic Exponential (ELLoGE) and Log-Logistic Exponential (LLoGE) distributions as well as the non-nested Gamma-Dagum (GD) [11, 13] distribution are presented.

The MLEs of the parameters (standard error in parenthesis), -2log-likelihood statistic $(-2 \ln (L))$, Akaike Information Criterion $(A I C=2 p-2 \ln (L))$, Bayesian Information Criterion $(B I C=p \ln (n)-2 \ln (L))$, and Corrected Akaike Information Criterion $\left(A I C C=A I C+2 \frac{p(p+1)}{n-p-1}\right)$, where $L=L(\hat{\Theta})$ is the value of the likelihood function evaluated at the parameter estimates, $n$ is the number of observations, and $p$ is the number of estimated parameters are presented. The goodness-of-fit statistics: Kolmogorov-Smirnov p-value (KS p-value), Cramer-von Mises $\left(W^{*}\right)$ and Anderson-Darling $\left(A^{*}\right)$ were also presented in the Tables. These statistics can be used to verify which distribution fits better to the data. In general, the smaller the values of $W^{*}$ and $A^{*}$, the better the fit. The ELLoGW distribution is fitted to the data sets and these fits are compared to the fits using exponentiated log-logistic exponential (ELLoGE), log-logistic Weibull (LLoGW) and log-logistic exponential (LLoGE) distributions. 
Fitted PDF

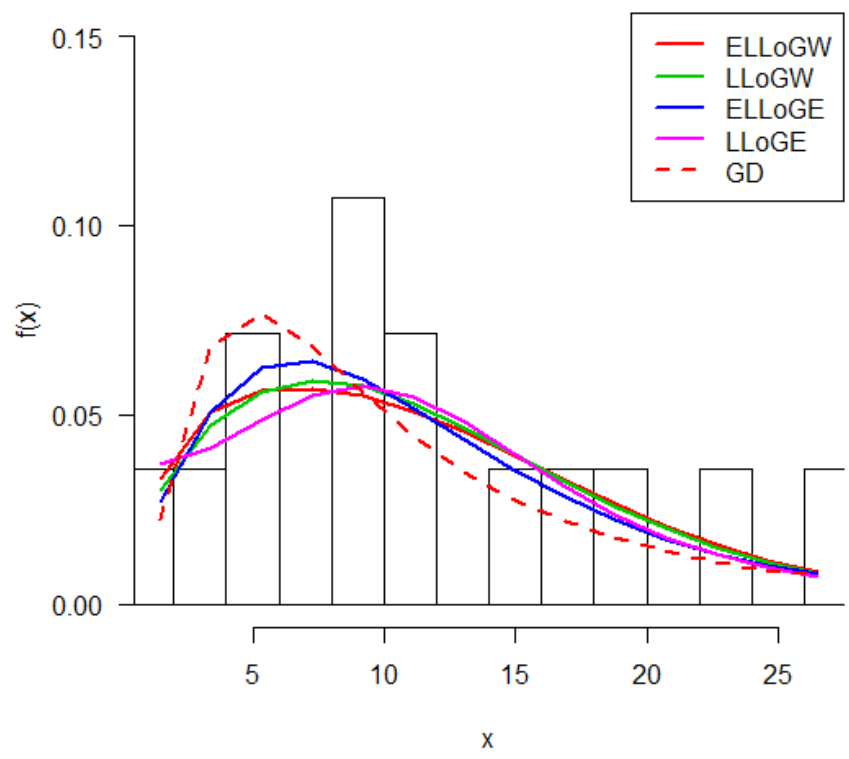

The Graph of Observed vs Expected Probability

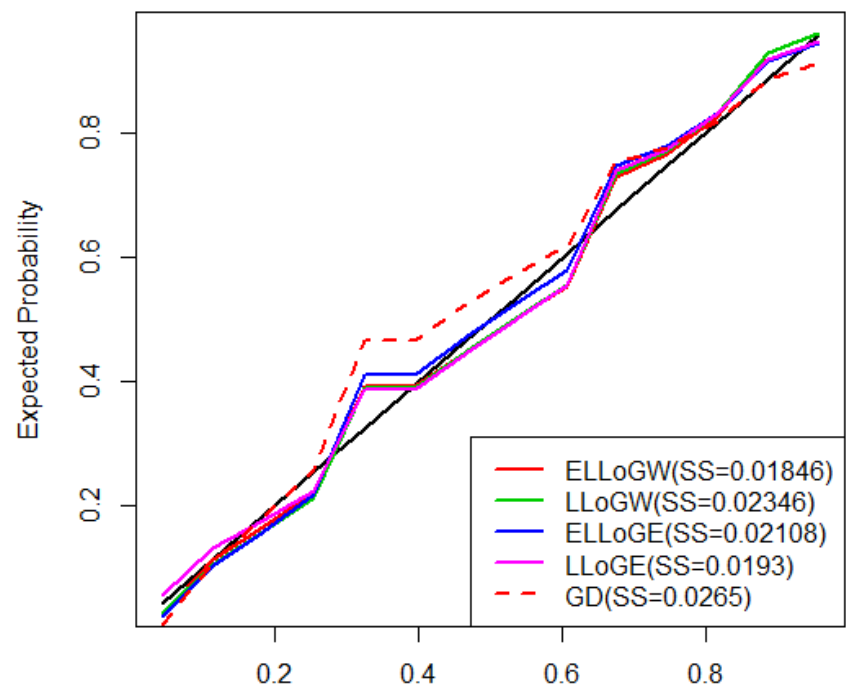

Observed Probability

Figure 1: Fitted Densities, Probability Plots for Times to Infection Data

Table 2: Maintenance Data: Type I right censoring

\begin{tabular}{|c|c|c|c|c|c|c|c|c|c|c|c|c|c|}
\hline \multirow[b]{2}{*}{ Model } & \multicolumn{5}{|c|}{ Estimates } & \multicolumn{8}{|c|}{ Statistics } \\
\hline & $s$ & $c$ & $\alpha$ & $\beta$ & $\delta$ & $-2 \log L$ & $A I C$ & AICC & $B I C$ & $W^{*}$ & $A^{*}$ & $K S p$-value & $S S$ \\
\hline ELLoGW & $\begin{array}{c}0.4681 \\
(0.4880)\end{array}$ & $\begin{array}{c}1.2222 \\
(0.2612)\end{array}$ & $\begin{array}{c}0.0023 \\
(0.0033)\end{array}$ & $\begin{array}{c}3.9238 \\
(0.8702)\end{array}$ & $\begin{array}{c}3.2765 \\
(2.9931)\end{array}$ & 115.3248 & 125.3248 & 127.2603 & 133.3794 & 0.0330 & 0.2262 & 0.8368 & 0.0360 \\
\hline LLoGW & $\begin{array}{c}1.7033 \\
(0.5094)\end{array}$ & $\begin{array}{c}1.4847 \\
(0.3395)\end{array}$ & $\begin{array}{c}0.0049 \\
(0.0301)\end{array}$ & $\begin{array}{c}3.4126 \\
(3.6607)\end{array}$ & $\begin{array}{l}1 \\
-\end{array}$ & 118.9152 & 126.9152 & 128.8507 & 133.3589 & 0.0744 & 0.4677 & 0.5637 & 0.0569 \\
\hline ELLoGE & $\begin{array}{c}0.0169 \\
(0.0207)\end{array}$ & $\begin{array}{c}0.6744 \\
(0.2535)\end{array}$ & $\begin{array}{c}0.4229 \\
(0.1848)\end{array}$ & $\begin{array}{l}1 \\
-\end{array}$ & $\begin{array}{l}27.9948 \\
(0.0003)\end{array}$ & 120.4324 & 128.4324 & 130.3678 & 134.8760 & 0.0576 & 0.3904 & 0.7290 & 0.0594 \\
\hline LLoGE & $\begin{array}{c}1.9005 \\
(1.1114)\end{array}$ & $\begin{array}{c}1.8526 \\
(0.3484)\end{array}$ & $\begin{array}{c}0.1235 \\
(0.2375)\end{array}$ & $\begin{array}{l}1 \\
-\end{array}$ & $\begin{array}{l}1 \\
-\end{array}$ & 124.1565 & 130.1565 & 132.0920 & 134.9893 & 0.0902 & 0.5814 & 0.6966 & 0.0641 \\
\hline GD & $\begin{array}{c}\lambda \\
1.7891 \\
(3.2287)\end{array}$ & $\begin{array}{c}\beta \\
2.6292 \\
(6.4012)\end{array}$ & $\begin{array}{c}\delta \\
0.4189 \\
(0.7477)\end{array}$ & $\begin{array}{c}\alpha \\
3.1506 \\
(8.2541)\end{array}$ & $\begin{array}{c}\theta \\
0.0332 \\
(0.1681)\end{array}$ & 121.6979 & 131.6979 & 133.6333 & 139.7525 & 0.0686 & 0.4541 & 0.6322 & 0.0724 \\
\hline
\end{tabular}

Table 3: Maintenance Data: Type II double censoring

\begin{tabular}{|c|c|c|c|c|c|c|c|c|c|c|c|c|c|}
\hline \multirow[b]{2}{*}{ Model } & \multicolumn{5}{|c|}{ Estimates } & \multicolumn{8}{|c|}{ Statistics } \\
\hline & $s$ & $c$ & $\alpha$ & $\beta$ & $\delta$ & $-2 \log L$ & $A I C$ & $A I C C$ & $B I C$ & $W^{*}$ & $A^{*}$ & $K S p-$ value & $S S$ \\
\hline ELLoGW & $\begin{array}{c}0.5157 \\
(0.1059)\end{array}$ & $\begin{array}{c}1.3934 \\
(0.2409)\end{array}$ & $\begin{array}{c}0.0003 \\
(0.0003)\end{array}$ & $\begin{array}{c}4.9765 \\
(0.7204)\end{array}$ & $\begin{array}{c}5.0079 \\
(0.0146)\end{array}$ & 97.5309 & 107.5309 & 109.9309 & 114.7009 & 0.0450 & 0.3378 & 0.5716 & 0.0535 \\
\hline LLoGW & $\begin{array}{l}21.0083 \\
(0.0046)\end{array}$ & $\begin{array}{c}1.6085 \\
(2.4384)\end{array}$ & $\begin{array}{c}0.1990 \\
(0.0849)\end{array}$ & $\begin{array}{c}1.6298 \\
(0.2486)\end{array}$ & $\begin{array}{l}1 \\
-\end{array}$ & 105.1903 & 113.1903 & 115.5903 & 118.9262 & 0.1017 & 0.6910 & 0.3093 & 0.0976 \\
\hline ELLoGE & $\begin{array}{c}0.0221 \\
(0.0337)\end{array}$ & $\begin{array}{c}0.9696 \\
(0.4440)\end{array}$ & $\begin{array}{c}0.3636 \\
(0.2369)\end{array}$ & $\begin{array}{l}1 \\
-\end{array}$ & $\begin{array}{c}98.3757 \\
(0.00007)\end{array}$ & 102.7356 & 110.7356 & 113.1356 & 116.4715 & 0.0777 & 0.5469 & 0.7063 & 0.0845 \\
\hline LLoGE & $\begin{array}{c}5.1884 \\
(0.3930)\end{array}$ & $\begin{array}{c}25.1414 \\
(26.2939)\end{array}$ & $\begin{array}{c}0.3791 \\
(0.0784)\end{array}$ & $\begin{array}{l}1 \\
-\end{array}$ & $\begin{array}{l}1 \\
-\end{array}$ & 106.8501 & 112.8501 & 115.2501 & 117.1521 & 0.0782 & 0.5817 & 0.1414 & 0.1575 \\
\hline GD & $\begin{array}{c}\lambda \\
1.7696 \\
(3.0503)\end{array}$ & $\begin{array}{c}\beta \\
2.9249 \\
(3.8793)\end{array}$ & $\begin{array}{c}\delta \\
0.5022 \\
(0.4692)\end{array}$ & $\begin{array}{c}\alpha \\
3.0936 \\
(4.9479)\end{array}$ & $\begin{array}{c}\theta \\
0.0341 \\
(0.0966)\end{array}$ & 104.1335 & 114.1335 & 116.5335 & 121.3034 & 0.0853 & 0.5947 & 0.5902 & 0.0895 \\
\hline
\end{tabular}

\subsection{Type I right censoring: Times to Infection Data}

The following example utilizes the times to infection (in months) of kidney dialysis patients with a surgically placed catheter. This data is given in Table 1.2 of Klein and Moeschberger [7]. The infection times are given in Table A.1 and the censored observations are given in Table A.2 in the appendix.

The initial values for the ELLoGW distribution in $\mathrm{R}$ code are $s=2, c=0.9, \alpha=0.01, \beta=1.9$ and $\delta=4$. The MLEs of the parameters of the ELLoGW distribution and its related submodels, AIC, AICC, BIC, $\mathrm{W}^{*}, \mathrm{~A}^{*}, \mathrm{KS}$ p-value and SS are given in Table 1, along with standard errors in parentheses. Plots of the fitted densities and the histogram, observed probability vs predicted probability ([6]) are given in Figure 1.

The likelihood ratio (LR) test statistic of the hypothesis $H_{0}$ : LLoGW against $H_{a}$ : ELLoGW and $H_{0}$ : ELLoGE against $H_{a}$ : ELLoGW are $0.166(\mathrm{p}$-value $=0.6837)$ and $0.4052(\mathrm{p}$-value 


\section{Fitted PDF}

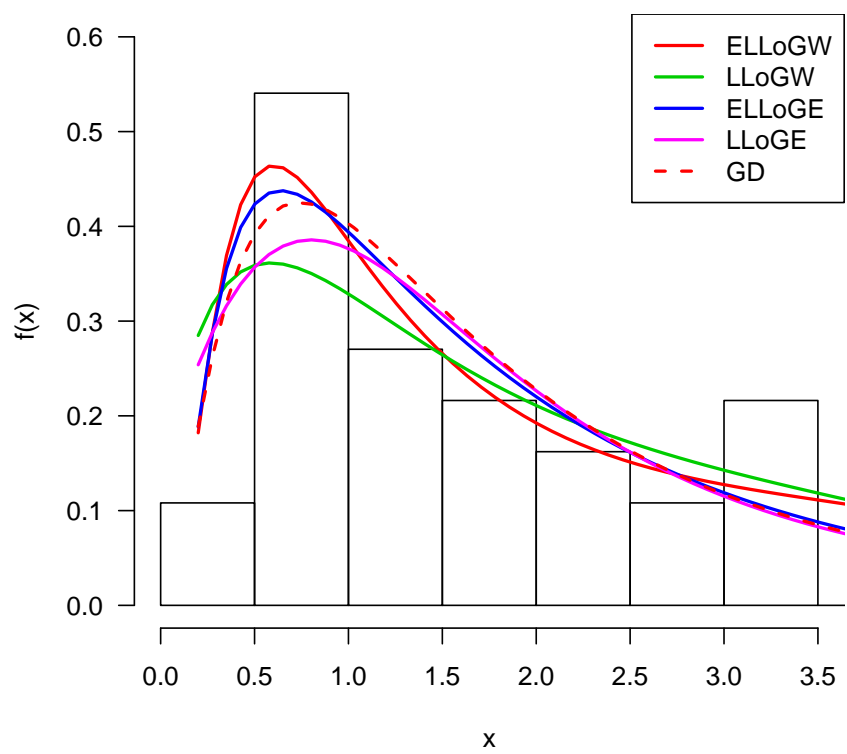

The Graph of Observed vs Expected Probability

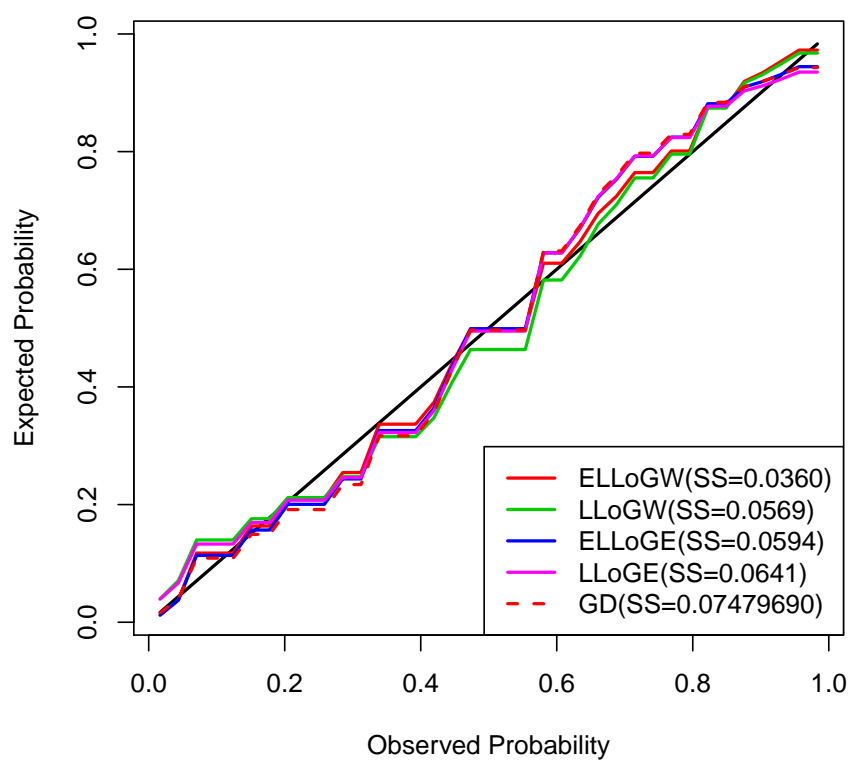

Figure 2: Fitted Densities, Probability Plots for Type I right censoring maintenance data

Fitted PDF

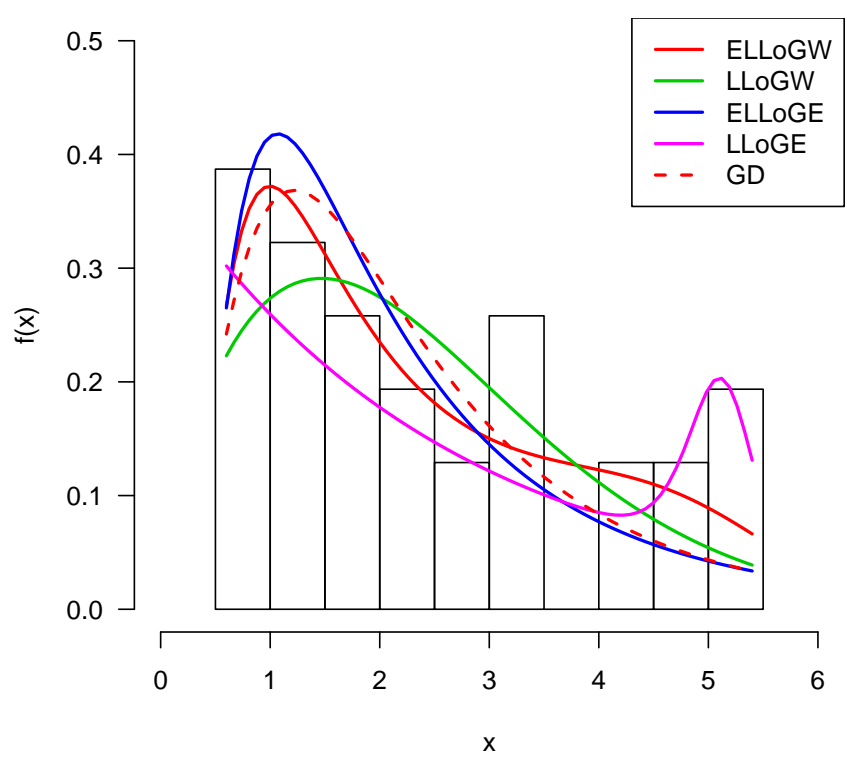

The Graph of Observed vs Expected Probability

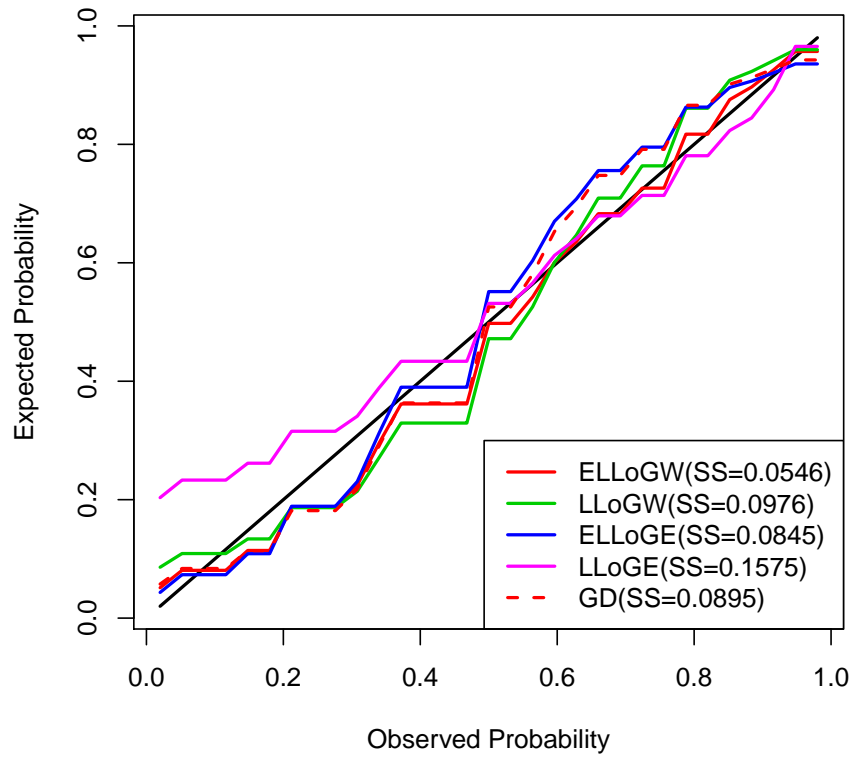

Figure 3: Fitted Densities, Probability Plots for Type II double censoring maintenance data

$=0.5244)$. We can conclude that there are no significant differences between fits of the LLoGW and ELLoGW distributions, as well as between fits of the ELLoGE and ELLoGW distributions. The values of the goodness-of-fit statistics $A^{*}$ and $W^{*}$ shows that the ELLoGW distribution is the better fit for the time to infection data. The Kolmogorov-Smirnov p-value shows that the ELLoGW distribution fits the data better than the other models. Also, the value of SS from the probability plot in Figure 1 is the smallest for ELLoGW model. Also, Figure 1 showed that the new model ELLoGW provided better fit for the density and probability plots than the LLOGW, ELLoGW, LLoGE and GD models. 


\subsection{Type I right censoring: Maintenance Data}

In the following example, we consider a maintenance data set. The set of data is the maintenance data with 46 observations reported on active repair times (hours) for an airborne communication transceiver. The data was discussed by Alven [8], Chhikara and Folks [9], and Dimitrakopoulou et al. [10]. The maintenance data set is given in Table A.3 in the appendix. The data given in Table A.4 has eight observations removed to illustrate type I right censoring (see appendix for the data).

Initial values for the ELLoGW model in R code are $s=0.3$, $c=1, \alpha=0.002, \beta=3$ and $\delta=3$. The MLEs of the parameters of the ELLoGW distribution and its related submodels, $\mathrm{AIC}, \mathrm{AICC}, \mathrm{BIC}, \mathrm{W}^{*}, \mathrm{~A}^{*}, \mathrm{KS}$ p-value and $\mathrm{SS}$ are given in Table 2. Plots of the fitted densities and the histogram, observed probability vs predicted probability are given in Figure 2.

The LR test statistic of the hypothesis $H_{0}$ : LLoGW against $H_{a}$ : ELLoGW and $H_{0}$ : ELLoGE against $H_{a}$ : ELLoGW are $3.5904(\mathrm{p}$-value $=0.0581)$ and $5.1076(\mathrm{p}$-value $=0.0238)$. We can conclude that there is a significant difference between the fits of the ELLoGE and ELLoGW distributions. There is also a significant difference between fits of the LLoGW and ELLoGW distributions at the $10 \%$ level of significance. The values of the goodness-of-fit statistics $A^{*}$ and $W^{*}$ shows that the ELLoGW distribution is by far the better fit for the maintenance data. The KS p-value shows that the ELLoGW distribution fits the maintenance data better than the other models. Figure 2 showed that the new model ELLoGW provided better fit for the density and probability plots than the LLOGW, ELLoGW, LLoGE and GD models.

\subsection{Type II double censoring: Maintenance Data}

In the following example we consider the maintenance data set with 15 observations removed to illustrate type II Double censoring. The data is given in Table A.5 in the appendix.

Initial values for the ELLoGW model are $s=0.4, c=1.4$, $\alpha=0.001, \beta=5$ and $\delta=5$. The MLEs of the parameters of the ELLoGW distribution and its related sub models, AIC, AICC, BIC, W*, A*, KS p-value and SS are given in Table 3, along with standard errors in parentheses. Plots of the fitted densities and the histogram, observed probability vs predicted probability are given in Figure 3.

The LR test statistic of the hypothesis $H_{0}$ : LLoGW against $H_{a}$ : ELLoGW and $H_{0}$ : ELLoGE against $H_{a}$ : ELLoGW are $7.6594(\mathrm{p}$-value $=0.0056)$ and $5.2047(\mathrm{p}$-value $=0.0225) . \mathrm{We}$ can conclude that there is a significant difference between the fits of the LLoGW and the ELLoGW distributions as well as between the fits of the ELLoGE and ELLoGW distributions. The values of the goodness-of-fit statistics $A^{*}$ and $W^{*}$ shows that the ELLoGW distribution is by far the better fit for the maintenance data. The KS p-value for the ELLoGW distribution is greater than that of the LLoGW and LLoGE models. Figure 3 showed that the new model ELLoGW provided better fit for the density and probability plots than the LLOGW, ELLoGW, LLoGE and GD models.

\section{Concluding Remarks}

We have presented the ELLoGW distribution under different censoring mechanisms. Applications of the model, under type I right censoring and type II double censoring, to real data are presented to illustrate its usefulness and applicability. The findings suggest that the ELLoGW distribution performed better than all other distributions considered.

\section{Acknowledgment}

We thank the referees for the positive enlightening comments and suggestions, which have greatly helped us in making improvements to this paper.

\section{References}

[1] B. O. Oluyede, G. Basele, S. Huang \& B. Makubate, "A New class of Generalized Log-Logistic Weibull Distribution: Theory, Properties and Applications", Journal of Probability and Statistical Sciences, 14 (2016) 171.

[2] D. Cox, "Regression Models and Life Tables (with Discussion)", Journal of the Royal Statistical Society, Series B 34 (1972) 187.

[3] P. Mdlongwa, B. O. Oluyede, A. K. A. Amey, A. F. Fagbamigbe \& B. Makubate, "Kumaraswamy Log-logistic Weibull Distribution: Model, Theory and Application to Lifetime and Survival Data", Heliyon 5 (2019) 1.

[4] E. T. Lee \& J. Wang, Statistical Methods for Survival Data Analysis, Wiley, New York, 1st Ed. (2003) 1

[5] B. O. Oluyede, B. Makubate, A. F. Fagbamigbe \& P. Mdlongwa, "A New Burr XII-Weibull-Logarithmic Distribution for Survival and Lifetime Data Analysis : Model, Theory \& Applications. Stats 1 (2018) 77.

[6] M. Pararai, G. Warahena-Liyanage \& B. O. Oluyede, "A New Class of Generalized Power Lindley Distribution with Applications to Lifetime Data", Theoretical Mathematics \& Applications 5 (2015) 53.

[7] J. P. Klein \& M. L. Moeschberger Survival Analysis Techniques for Censored and Truncated Data, Springer-Verlag, New York, 2003.

[8] W.H. Alven Reliability Engineering by ARINC, Prentice-Hall, New Jersey, 1964.

[9] R. S. Chikara \& J. L. Folks, "The Inverse Gaussian Distribution as a Lifetime Model", Technometrics 19 (1977) 461.

[10] T. Dimitrakopoulou, K. Adamidis \& S. Loukas, "A Lifetime Distribution with an Upside-Down Bathtub-Shaped Hazard Function", IEEE Transactions on Reliability 56 (2007) 308.

[11] B. O. Oluyede \& S. Huang, "Estimation in the Exponentiated Kumaraswamy Dagum Distribution with Censored Samples", Electronic Journal of Applied Statistical Analysis 8 (2016) 122.

[12] T. S. Ferguson, A Course in Large Sample Theory, Chapman \& Hall (1996).

[13] B. O. Oluyede, S. Huang \& M. Pararai, "A New Class of Generalized Dagum Distribution with Applications to Income and Lifetime Data", Journal of Statistical and Econometric Methods 3 (2014) 125. 
Fagbamigbe et al. / J. Nit. Soc. Phys. Sci. 1 (2019) 12-19

18

Appendix A. Elements of the Score Vector: Type I Right Censoring

Elements of the score vector for the Type I right censoring scheme are given by,

$$
\begin{aligned}
& \frac{\partial \ell(\underline{\Theta})}{\partial s}=\sum_{i=1}^{n}\left\{\epsilon _ { i } \left[\delta(\delta-1)\left(1-\left(1+\left(\frac{t_{i}}{s}\right)^{c}\right)^{-1} e^{-\alpha t_{i}^{\beta}}\right)^{\delta-2}(-1)\left(1+\left(\frac{t_{i}}{s}\right)\right)^{-3}\left(\frac{t_{i}}{s}\right)^{c-1}\left(\frac{c t_{i}}{s^{2}}\right) e^{-\alpha t_{i}^{\beta}}\left(\alpha \beta t_{i}^{\beta-1}+\frac{c t_{i}^{c-1}}{s^{c}+t_{i}^{c}}\right)\right.\right. \\
& \left.+\left[\delta\left(1-\left(1+\left(\frac{t_{i}}{s}\right)^{c}\right)^{-1} e^{-\alpha t_{i}^{\beta}}\right)^{\delta-1} e^{-\alpha t_{i}^{\beta}}\right]\left[\left(1+\left(\frac{t_{i}}{s}\right)^{c}\right)^{-2}\left(\frac{t_{i}}{s}\right)^{c-1}\left(\frac{c t_{i}}{s^{2}}\right)\left(\alpha \beta t_{i}^{\beta-1}+\frac{c t_{i}^{c-1}}{s^{c}+t_{i}^{c}}\right)-\left(1+\left(\frac{t_{i}}{s}\right)^{c}\right)^{-1} \frac{c^{2}\left(s t_{i}\right)^{c-1}}{\left(s^{c}+t_{i}^{c}\right)^{2}}\right]\right] \\
& \times\left[\delta\left(1-\left(1+\left(\frac{t_{i}}{s}\right)^{c}\right)^{-1} e^{-\alpha t_{i}^{\beta}}\right)^{\delta-1} e^{-\alpha t_{i}^{\beta}}\left(1+\left(\frac{t_{i}}{s}\right)\right)^{-1}\left(\alpha \beta t_{i}^{\beta-1}+\frac{c t_{i}^{c-1}}{s^{c}+t_{i}^{c}}\right)\right]^{-1} \\
& \left.+\left(1-\epsilon_{i}\right)\left[\delta\left(1-\left(1+\left(\frac{t_{i}}{s}\right)^{c}\right)^{-1} e^{-\alpha t_{i}^{\beta}}\right)^{\delta-1} e^{-\alpha t_{i}^{\beta}}\left(1+\left(\frac{t_{i}}{s}\right)^{c}\right)^{-2}\left(\frac{t_{i}}{s}\right)^{c-1}\left(\frac{c t_{i}}{s^{2}}\right)\right]\left[1-\left(1-\left(1+\left(\frac{t_{i}}{s}\right)^{c}\right)^{-1} e^{-\alpha t_{i}^{\beta}}\right)^{\delta}\right]^{-1}\right\}, \\
& \frac{\partial \ell(\underline{\Theta})}{\partial c}=\sum_{i=1}^{n}\left\{\epsilon _ { i } \left[\delta(\delta-1)\left(1-\left(1+\left(\frac{t_{i}}{s}\right)^{c}\right)^{-1} e^{-\alpha t_{i}^{\beta}}\right)^{\delta-2}(-1)\left(1+\left(\frac{t_{i}}{s}\right)\right)^{-3}\left(\frac{t_{i}}{s}\right)^{c} \ln \left(\frac{t_{i}}{s}\right) e^{-\alpha t_{i}^{\beta}}\left(\alpha \beta t_{i}^{\beta-1}+\frac{c t_{i}^{c-1}}{s^{c}+t_{i}^{c}}\right)\right.\right. \\
& +\left[\delta\left(1-\left(1+\left(\frac{t_{i}}{s}\right)^{c}\right)^{-1} e^{-\alpha t_{i}^{\beta}}\right)^{\delta-1} e^{-\alpha t_{i}^{\beta}}\right]\left[(-1)\left(1+\left(\frac{t_{i}}{s}\right)^{c}\right)^{-1}\left(\frac{t_{i}}{s}\right)^{c} \ln \left(\frac{t_{i}}{s}\right)\left(\alpha \beta t_{i}^{\beta-1}+\frac{c t_{i}^{c-1}}{s^{c}+t_{i}^{c}}\right)-\left(1+\left(\frac{t_{i}}{s}\right)^{c}\right)^{-1}\right. \\
& \left.\times \frac{t_{i}^{2(c-1)} \ln \left(t_{i}\right)\left(s^{c}+t_{i}^{c}\right)-c t_{i}^{c-1}\left(s^{c} \ln s+t_{i}^{c} \ln t_{i}\right)}{\left(s^{c}+t_{i}^{c}\right)^{2}}\right]\left[\delta\left(1-\left(1+\left(\frac{t_{i}}{s}\right)^{c}\right)^{-1} e^{-\alpha t_{i}^{\beta}}\right)^{\delta-1} e^{-\alpha t_{i}^{\beta}}\left(1+\left(\frac{t_{i}}{s}\right)\right)^{-1}\left(\alpha \beta t_{i}^{\beta-1}+\frac{c t_{i}^{c-1}}{s^{c}+t_{i}^{c}}\right)\right]^{-1} \\
& \left.+\left(1-\epsilon_{i}\right)\left[\delta\left(1-\left(1+\left(\frac{t_{i}}{s}\right)^{c}\right)^{-2} e^{-\alpha t_{i}^{\beta}}\right)^{\delta-1} e^{-\alpha t_{i}^{\beta}}\left(1+\left(\frac{t_{i}}{s}\right)^{c}\right)^{-2}\left(\frac{t_{i}}{s}\right)^{c} \ln \left(\frac{t_{i}}{s}\right)\right]\left[1-\left(1-\left(1+\left(\frac{t_{i}}{s}\right)^{c}\right)^{-1} e^{-\alpha t_{i}^{\beta}}\right)^{\delta}\right]^{-1}\right\} \\
& \frac{\partial \ell \underline{(\Theta)}}{\partial \alpha}=\sum_{i=1}^{n}\left\{\epsilon _ { i } \left[\left[\delta(\delta-1)\left(1-\left(1+\left(\frac{t_{i}}{s}\right)^{c}\right)^{-1} e^{-\alpha t_{i}^{\beta}}\right)^{\delta-2}\left(1+\left(\frac{t_{i}}{s}\right)\right)^{-1} t_{i}^{\beta} e^{-2 \alpha t_{i}^{\beta}}-\delta\left(1-\left(1+\left(\frac{t_{i}}{s}\right)^{c}\right)^{-1} e^{-\alpha t_{i}^{\beta}}\right)^{\delta-1}\left(1+\left(\frac{t_{i}}{s}\right)\right)^{-1} t_{i}^{\beta} e^{-\alpha t_{i}^{\beta}}\right]\right.\right. \\
& \left.\times\left(1+\left(\frac{t_{i}}{s}\right)\right)^{-1}\left(\alpha \beta t_{i}^{\beta-1}+\frac{c t_{i}^{c-1}}{s^{c}+t_{i}^{c}}\right)+\delta\left(1-\left(1+\left(\frac{t_{i}}{s}\right)^{c}\right)^{-1} e^{-\alpha t_{i}^{\beta}}\right)^{\delta-1} e^{-\alpha t_{i}^{\beta}}\left(1+\left(\frac{t_{i}}{s}\right)^{c}\right)^{-1} \beta t_{i}^{\beta-1}\right] \\
& \times\left[\delta\left(1-\left(1+\left(\frac{t_{i}}{s}\right)^{c}\right)^{-1} e^{-\alpha t_{i}^{\beta}}\right)^{\delta-1} e^{-\alpha t_{i}^{\beta}}\left(1+\left(\frac{t_{i}}{s}\right)\right)^{-1}\left(\alpha \beta t_{i}^{\beta-1}+\frac{c t_{i}^{c-1}}{s^{c}+t_{i}^{c}}\right)\right]^{-1}+\left(1-\epsilon_{i}\right)\left[\delta\left(1-\left(1+\left(\frac{t_{i}}{s}\right)^{c}\right)^{-1} e^{-\alpha t_{i}^{\beta}}\right)^{\delta-1}\right. \\
& \left.\left.\times e^{-\alpha t_{i}^{\beta}}\left(1+\left(\frac{t_{i}}{s}\right)^{c}\right)^{-1}\left(-t_{i}^{\beta}\right)\right]\left[1-\left(1-\left(1+\left(\frac{t_{i}}{s}\right)^{c}\right)^{-1} e^{-\alpha t_{i}^{\beta}}\right)^{\delta}\right]^{-1}\right\} \\
& \frac{\partial \ell(\underline{\Theta})}{\partial \beta}=\sum_{i=1}^{n}\left\{\epsilon _ { i } \left[\left[\delta(\delta-1)\left(1-\left(1+\left(\frac{t_{i}}{s}\right)^{c}\right)^{-1} e^{-\alpha t_{i}^{\beta}}\right)^{\delta-2}\left(1+\left(\frac{t_{i}}{s}\right)\right)^{-1} \alpha t_{i}^{\beta} e^{-2 \alpha t_{i}^{\beta}} \ln \left(t_{i}\right)-\delta\left(1-\left(1+\left(\frac{t_{i}}{s}\right)^{c}\right)^{-1} e^{-\alpha t_{i}^{\beta}}\right)^{\delta-1}\left(1+\left(\frac{t_{i}}{s}\right)\right)^{-1}\right.\right.\right. \\
& \left.\left.\times \alpha t_{i}^{\beta} e^{-\alpha t_{i}^{\beta}} \ln \left(t_{i}\right)\right]\left(1+\left(\frac{t_{i}}{s}\right)\right)^{-1}\left(\alpha \beta t_{i}^{\beta-1}+\frac{c t_{i}^{c-1}}{s^{c}+t_{i}^{c}}\right)+\delta\left(1-\left(1+\left(\frac{t_{i}}{s}\right)^{c}\right)^{-1} e^{-\alpha t_{i}^{\beta}}\right)^{\delta-1} e^{-\alpha t_{i}^{\beta}}\left(1+\left(\frac{t_{i}}{s}\right)^{c}\right)^{-1}\left(\alpha t_{i}^{\beta-1}+\alpha \beta t_{i}^{\beta-1} \ln \left(t_{i}\right)\right)\right] \\
& \times\left[\delta\left(1-\left(1+\left(\frac{t_{i}}{s}\right)^{c}\right)^{-1} e^{-\alpha t_{i}^{\beta}}\right)^{\delta-1} e^{-\alpha t_{i}^{\beta}}\left(1+\left(\frac{t_{i}}{s}\right)\right)^{-1}\left(\alpha \beta t_{i}^{\beta-1}+\frac{c t_{i}^{c-1}}{s^{c}+t_{i}^{c}}\right)\right]^{-1}+\left(1-\epsilon_{i}\right)\left[\delta\left(1-\left(1+\left(\frac{t_{i}}{s}\right)^{c}\right)^{-1} e^{-\alpha t_{i}^{\beta}}\right)^{\delta-1}\right. \\
& \left.\left.\times e^{-\alpha t_{i}^{\beta}}\left(1+\left(\frac{t_{i}}{s}\right)^{c}\right)^{-1}\left(-\alpha t_{i}^{\beta}\right) \ln \left(t_{i}\right)\right]\left[1-\left(1-\left(1+\left(\frac{t_{i}}{s}\right)^{c}\right)^{-1} e^{-\alpha t_{i}^{\beta}}\right)^{\delta}\right]^{-1}\right\}
\end{aligned}
$$

and

$$
\begin{aligned}
\frac{\partial \ell(\underline{\Theta})}{\partial \delta}= & \sum_{i=1}^{n}\left\{\epsilon _ { i } \left[\delta\left(1-\left(1+\left(\frac{t_{i}}{s}\right)^{c}\right)^{-1} e^{-\alpha t_{i}^{\beta}}\right)^{\delta-1} e^{-\alpha t_{i}^{\beta}} \ln \left(1-\left(1+\left(\frac{t_{i}}{s}\right)^{c}\right)^{-1} e^{-\alpha t_{i}^{\beta}}\right)+\left(1-\left(1+\left(\frac{t_{i}}{s}\right)^{c}\right)^{-1} e^{-\alpha t_{i}^{\beta}}\right]\right.\right. \\
& \times\left[\delta\left(1-\left(1+\left(\frac{t_{i}}{s}\right)^{c}\right)^{-1} e^{-\alpha t_{i}^{\beta}}\right)^{\delta-1} e^{-\alpha t_{i}^{\beta}}\left(1+\left(\frac{t_{i}}{s}\right)\right)^{-1}\left(\alpha \beta t_{i}^{\beta-1}+\frac{c t_{i}^{c-1}}{s^{c}+t_{i}^{c}}\right)\right]^{-1}+\left(1-\epsilon_{i}\right)\left[\left(1-\left(1+\left(\frac{t_{i}}{s}\right)^{c}\right)^{-1} e^{-\alpha t_{i}^{\beta}}\right)^{\delta}\right. \\
& \left.\left.\times \ln \left(1-\left(1+\left(\frac{t_{i}}{s}\right)^{c}\right)^{-1} e^{-\alpha t_{i}^{\beta}}\right)\right]\left[1-\left(1-\left(1+\left(\frac{t_{i}}{s}\right)^{c}\right)^{-1} e^{-\alpha t_{i}^{\beta}}\right)^{\delta}\right]^{-1}\right\} .
\end{aligned}
$$

18 


\section{Tables with Complete and Censored data}

The infection times are given in Table A.1 and the censored observations are given in Table A.2. The complete and censored maintenance data are given in Tables A.3, A.4 and A.5, respectively.

Table A.1: Infection Times

\begin{tabular}{rrrrrr}
\hline 1.5 & 3.5 & 4.5 & 5.5 & 8.5 & 8.5 \\
10.5 & 11.5 & 15.5 & 16.5 & 18.5 & 2.5 \\
\hline
\end{tabular}

Table A.2: Censored Infection Times

\begin{tabular}{|c|c|c|c|c|c|c|}
\hline 2.5 & 2.5 & 3.5 & 3.5 & 3.5 & 4.5 & 4.5 \\
\hline 5.5 & 6.5 & 6.5 & 7.5 & 7.5 & 7.5 & 7.5 \\
\hline 8.5 & 9.5 & 10.5 & 11.5 & 12.5 & 12.5 & 13.5 \\
\hline 14.5 & 14.5 & 21.5 & 21.5 & 22.5 & 22.5 & 25.5 \\
\hline
\end{tabular}

Table A.3: Complete Maintenance Data

\begin{tabular}{rrrrrrrrrrr}
\hline 0.2 & 0.3 & 0.5 & 0.5 & 0.5 & 0.5 & 0.6 & 0.6 & 0.7 & 0.7 & 0.7 \\
0.8 & 0.8 & 1.0 & 1.0 & 1.0 & 1.0 & 1.1 & 1.3 & 1.5 & 1.5 & \\
1.5 & 2.0 & 2.0 & 2.2 & 2.5 & 2.7 & 3.0 & 3.0 & 3.3 & 3.3 \\
4.0 & 4.5 & 4.7 & 5.0 & 5.4 & 5.4 & 7.0 & 7.5 & 8.8 & 9.0 \\
22.0 & 24.5 & & & & & & & & & \\
\hline
\end{tabular}

Table A.4: Type I Right Censored Maintenance Data

\begin{tabular}{llllllllll}
\hline 0.2 & 0.3 & 0.5 & 0.5 & 0.5 & 0.5 & 0.6 & 0.6 & 0.7 & 0.7 \\
0.8 & 0.8 & 1.0 & 1.0 & 1.0 & 1.1 & 1.3 & 1.5 & 1.5 & 1.5 \\
2.0 & 2.0 & 2.2 & 2.5 & 2.7 & 3.0 & 3.0 & 3.3 & 3.3 & 4.0 \\
4.5 & 4.7 & 5.0 & 5.4 & 5.4 & & & & & \\
\hline
\end{tabular}

Table A.5: Maintenance Data II

\begin{tabular}{llllllllll}
\hline 0.6 & 0.7 & 0.7 & 0.7 & 0.8 & 0.8 & 1.0 & 1.0 & 1.0 & 1.1 \\
1.5 & 1.5 & 1.5 & 1.5 & 2.0 & 2.0 & 2.2 & 2.5 & 2.7 & 3.0 \\
3.3 & 3.3 & 4.0 & 4.0 & 4.5 & 4.7 & 5.0 & 5.4 & 5.4 & \\
\hline
\end{tabular}

